\title{
Iterative approach of 3D datum transformation with a non-isotropic weight
}

\author{
Huaien Zeng ${ }^{1,2,3} \cdot$ Qinglin $\mathrm{Yi}^{2} \cdot$ Yue $\mathrm{Wu}^{2}$
}

Received: 11 July 2015/Accepted: 26 September 2015/Published online: 8 October 2015

(C) Akadémiai Kiadó 2015

\begin{abstract}
The analytical solution of 3D datum transformation with an isotropic weight has been elegantly presented based on Procrustes algorithm (singular value decomposition). But the existence of analytical solution of 3D datum transformation with a nonisotropic weight needs further investigation. Based on the Lagrangian extremum law, the paper derives the analytical formula for translation parameter and scale factor, but because the rotation matrix is unsolved, the analytical solution does not exist. For this reason, the paper presents two kinds of iterative approach of 3D datum transformation with a nonisotropic weight. One is the iterative approach dependent on the objective function value, which uses the Lagrangian minimum function in the variable of rotation matrix as the objective function, and the other is the iterative approach dependent on the derivative of function, which uses the $3 \mathrm{D}$ datum transformation model that eliminates the translation parameter. In order to improve the speed and reliability of iterative computation, the form of rotation matrix represented by Rodrigues matrix instead of rotation angles or unit quaternion is adopted for the two iterative approaches. A numerical experiment is demonstrated, and comparison analysis of the two iterative approaches is carried out. The result shows from the view of computing speed and reliability, the iterative approach based on derivatives is preferred.
\end{abstract}

Keywords 3D datum transformation - Lagrangian extremum - Non-isotropic weight . Iterative approach · Procrustes algorithm

Huaien Zeng

zenghuaien_2003@163.com

1 Hubei Key Laboratory of Construction and Management in Hydropower Engineering, China Three Gorges University, Yichang 443002, China

2 Key Laboratory of Geological Hazards on Three Gorges Reservoir Area (China Three Gorges University), Ministry of Education, Yichang 443002, China

3 Hubei Key Laboratory of Intelligent Vision Based Monitoring for Hydroelectric Engineering, China Three Gorges University, Yichang 443002, China 


\section{Introduction}

Three-dimensional datum transformation is a central problem in geodesy, engineering surveying, photogrammetry, geographical information science (GIS), etc., e.g. Aktuğ (2012), Akyilmaz (2007), Burša (1967), Chen et al. (2004), Dermanis (1998), El-Mowafy et al. (2009), Ge et al. (2013), Kashani (2006), Neitzel (2010), Paláncz et al. (2013), Soler and Snay (2004), Soycan and Soycan (2008), Watson (2006), Zeng (2014, 2015). It aims to determine the transformation parameter of the transformation model. Usually, the transformation model adopts seven-parameter similarity transformation, including three translation parameters and three rotation angle parameters and one scale factor, see Leick (2004), Leick and van Gelder (1975). So far a lot of approaches for computing the seven parameters have been presented, which can be classified into two categories. One is analytical algorithm, and the other one is iterative algorithm. The former can give the exact solution quickly by the analytical formulae of the parameters. Its advantage over the latter is that it does not need the initial parameter values as well as iterative computation. Due to the difficulty of mathematical derivation, rare analytical algorithms have been put forward. E.g., the most famous analytical algorithm is the Procrustes algorithm presented by Grafarend and Awange (2003). It used the singular value decomposition technique to solve the unconstrained Lagrangian extremum problem, and obtained the computation of rotation matrix. It can deal with the case that the weight is isotropic, namely the weight is identical in different coordinate direction. Shen et al. (2006) presented a quaternion-based algorithm by means of eigenvalue-eigenvector decomposition. Han (2010) presented a step-wise approach to compute the transformation parameters considering the physical meaning of similarity transformation. Zeng and Yi (2010a) presented an analytical algorithm based on Rodrigues matrix. The latter needs the initial parameter values, and iterative computation. Although it has the initial values problem of parameter, i.e. in the case that the rotation angle is large, the iterative computation fails due to a bad initial value of parameter (see e.g. Zeng and Tao 2003; Zeng and Yi 2011), it plays the dominant role at present, which is proved by a large amount of literature published recently. E.g., Zeng and Tao (2003) investigated the feasibility of linearization of transformation model in different rotation angles. Zeng and Huang (2008) presented a kind of searching method based on genetic algorithm and pattern search method that is suitable for any size of rotation angle. ElHabiby, et al. (2009) compared four non-linear least squares methods for 3D coordinates transformation, namely Steepest Descent, Trust region, Gauss-Newton and LevenbergMarquardt. Zeng and Yi (2011) presented a quaternion-based iterative solution of 3D coordinates transformation. It overcomes the initial value problem of parameter and is valid for 3D coordinate transformation of any rotation angle.

This paper is organized as follows. In Sect. 2, the analytical solution of 3D datum transformation with a non-isotropic weight is investigated in detail based on Lagrangian extremum law. The analytical formulae of translation parameter and scale factor are obtained, but the rotation matrix is unsolved, i.e. the analytical solution does not exist. For this reason, two kinds of iterative approaches are presented. One is the iterative approach dependent on the objective function value introduced in Sect. 3, which uses the Lagrangian minimum function as the objective function, and the other is the iterative approach dependent on the derivative of function introduced in Sect. 4, which uses the 3D datum transformation model that eliminates the translation parameter. In Sect. 5, a numerical experiment is given to demonstrate the presented two approaches, and comparisons of the two approaches are carried out. Lastly conclusions are made in the last section, i.e. Sect. 6. 


\section{Basic mathematical model of 3D datum transformation with a non- isotropic weight}

The seven-parameter similarity transformation model can be expressed as

$$
A_{i}=\lambda R B_{i}+t
$$

subject to

$$
R^{T} R=I, \operatorname{det}(R)=+1,
$$

where $A_{i}=\left[\begin{array}{lll}X_{i} & Y_{i} & Z_{i}\end{array}\right]^{T}$ and $B_{i}=\left[\begin{array}{lll}x_{i} & y_{i} & z_{i}\end{array}\right]^{T}(i=1,2, \cdots, n)$ are the 3D coordinates of a control point in the target and source coordinate systems of transformation, labelled as system A and system B respectively. Superscript $T$ represents transpose, $I$ denotes an identity matrix with the dimension of three, det is the determinant computation of matrix. $\lambda$ denotes the scale factor, $t=\left[\begin{array}{lll}\Delta X & \Delta Y & \Delta Z\end{array}\right]^{T}$ denotes the three translation parameters, and $R$ denotes the rotation matrix.

The computation problem of transformation parameters is sought in the principle of least squares, namely

$$
L(t, \lambda, \mathrm{R})=\sum_{i=1}^{n}\left(A_{i}-\lambda R B_{i}-t\right)^{T} P_{i}\left(A_{i}-\lambda R B_{i}-t\right)=\min ,
$$

where $P_{i}$ is the weight matrix corresponding to the control point $i$, which is non-isotropic and even correlated in different coordinate direction.

Equation (3) is essentially an optimization problem, and can usually be solved by the means of Lagrangian extremum law. If and only if the following conditions are satisfied, the Lagrangian extremum exists.

$$
\begin{aligned}
& \frac{\partial \mathrm{L}}{\partial t}=0, \\
& \frac{\partial \mathrm{L}}{\partial \lambda}=0, \\
& \frac{\partial \mathrm{L}}{\partial R}=0 .
\end{aligned}
$$

By Eqs. (3) and (4), one gets

$$
\frac{\partial L}{\partial t}=\sum_{i=1}^{n}-2\left(A_{i}-\lambda R B_{i}-t\right)^{T} P_{i}=0,
$$

further making the transpose of Eq. (7), one gets

$$
\sum_{i=1}^{n} P_{i}^{T}\left(A_{i}-\lambda R B_{i}-t\right)=0
$$

and then

$$
t=\left(\sum_{i=1}^{n} P_{i}^{T}\right)^{-1} \sum_{i=1}^{n} P_{i}^{T}\left(A_{i}-\lambda R B_{i}\right)
$$


Let

$$
p_{i}=\left(\sum_{i=1}^{n} P_{i}^{T}\right)^{-1} P_{i}^{T},
$$

thus

$$
t=\sum_{i=1}^{n} p_{i}\left(A_{i}-\lambda R B_{i}\right)
$$

Obviously, $t$ is the function form of $\lambda$ and $R$.

Substituting Eq. (11) into Eq. (3), one gets

$$
\begin{aligned}
L(\lambda, \mathrm{R})= & \sum_{i=1}^{n}\left(A_{i}-\lambda R B_{i}-\sum_{j=1}^{n} p_{j}\left(A_{j}-\lambda R B_{j}\right)\right)^{T} P_{i}\left(A_{i}-\lambda R B_{i}-\sum_{j=1}^{n} p_{j}\left(A_{j}-\lambda R B_{j}\right)\right) \\
= & \sum_{i=1}^{n}\left(A_{i}-\sum_{j=1}^{n} p_{j} A_{j}-\lambda\left(R B_{i}-\sum_{j=1}^{n} p_{j} R B_{j}\right)\right)^{T} \\
& \times P_{i}\left(A_{i}-\sum_{j=1}^{n} p_{j} A_{j}-\lambda\left(R B_{i}-\sum_{j=1}^{n} p_{j} R B_{j}\right)\right) .
\end{aligned}
$$

Let

$$
\bar{A}_{i}=A_{i}-\sum_{j=1}^{n} p_{j} A_{j},
$$

thus Eq. (12) is written as

$$
\begin{aligned}
L(\lambda, \mathrm{R})= & \sum_{i=1}^{n}\left(\bar{A}_{i}-\lambda\left(R B_{i}-\sum_{j=1}^{n} p_{j} R B_{j}\right)\right)^{T} P_{i}\left(\bar{A}_{i}-\lambda\left(R B_{i}-\sum_{j=1}^{n} p_{j} R B_{j}\right)\right) \\
= & \sum_{i=1}^{n}\left(\begin{array}{c}
\left.\bar{A}_{i}^{T} P_{i} \bar{A}_{i}-\lambda \bar{A}_{i}^{T} P_{i}\left(R B_{i}-\sum_{j=1}^{n} p_{j} R B_{j}\right)-\lambda\left(R B_{i}-\sum_{j=1}^{n} p_{j} R B_{j}\right)^{T} P_{i} \bar{A}_{i}\right) \\
+\lambda^{2}\left(R B_{i}-\sum_{j=1}^{n} p_{j} R B_{j}\right)^{T} P_{i}\left(R B_{i}-\sum_{j=1}^{n} p_{j} R B_{j}\right)
\end{array}\right) \\
= & \sum_{i=1}^{n}\left(\begin{array}{c}
\bar{A}_{i}^{T} P_{i} \bar{A}_{i}-\lambda \bar{A}_{i}^{T} P_{i}\left(R B_{i}-\sum_{j=1}^{n} p_{j} R B_{j}\right)-\lambda \bar{A}_{i}^{T} P_{i}^{T}\left(R B_{i}-\sum_{j=1}^{n} p_{j} R B_{j}\right) \\
+\lambda^{2}\left(R B_{i}-\sum_{j=1}^{n} p_{j} R B_{j}\right)^{T} P_{i}\left(R B_{i}-\sum_{j=1}^{n} p_{j} R B_{j}\right)
\end{array}\right) \\
= & \sum_{i=1}^{n}\left(\begin{array}{c}
\bar{A}_{i}^{T} P_{i} \bar{A}_{i}-\lambda \bar{A}_{i}^{T}\left(P_{i}+P_{i}^{T}\right)\left(R B_{i}-\sum_{j=1}^{n} p_{j} R B_{j}\right) \\
+\lambda^{2}\left(R B_{i}-\sum_{j=1}^{n} p_{j} R B_{j}\right)^{T} P_{i}\left(R B_{i}-\sum_{j=1}^{n} p_{j} R B_{j}\right)
\end{array}\right) .
\end{aligned}
$$


Substituting Eq. (14) into Eq. (5), one gets

$$
\begin{aligned}
\frac{\partial \mathrm{L}}{\partial \lambda}= & -\sum_{i=1}^{n}\left(\bar{A}_{i}^{T}\left(P_{i}+P_{i}^{T}\right)\left(R B_{i}-\sum_{j=1}^{n} p_{j} R B_{j}\right)\right) \\
& +2 \lambda \sum_{i=1}^{n}\left(\left(R B_{i}-\sum_{j=1}^{n} p_{j} R B_{j}\right)^{T} P_{i}\left(R B_{i}-\sum_{j=1}^{n} p_{j} R B_{j}\right)\right)=0,
\end{aligned}
$$

thus

$$
\lambda=\frac{\sum_{i=1}^{n}\left(\bar{A}_{i}^{T}\left(P_{i}+P_{i}^{T}\right)\left(R B_{i}-\sum_{j=1}^{n} p_{j} R B_{j}\right)\right)}{2 \sum_{i=1}^{n}\left(\left(R B_{i}-\sum_{j=1}^{n} p_{j} R B_{j}\right)^{T} P_{i}\left(R B_{i}-\sum_{j=1}^{n} p_{j} R B_{j}\right)\right)} .
$$

Obviously, $\lambda$ is the function form of $R$.

Substituting Eq. (16) into Eq. (14), one gets

$$
\begin{aligned}
L(\mathrm{R})= & \sum_{i=1}^{n}\left(\bar{A}_{i}^{T} P_{i} \bar{A}_{i}\right)-\frac{\sum_{i=1}^{n}\left(\bar{A}_{i}^{T}\left(P_{i}+P_{i}^{T}\right)\left(R B_{i}-\sum_{j=1}^{n} p_{j} R B_{j}\right)\right)}{2 \sum_{i=1}^{n}\left(\left(R B_{i}-\sum_{j=1}^{n} p_{j} R B_{j}\right)^{T} P_{i}\left(R B_{i}-\sum_{j=1}^{n} p_{j} R B_{j}\right)\right)} \\
& \times \sum_{i=1}^{n}\left(\bar{A}_{i}^{T}\left(P_{i}+P_{i}^{T}\right)\left(R B_{i}-\sum_{j=1}^{n} p_{j} R B_{j}\right)\right) \\
& \left.\left.+\frac{\sum_{i=1}^{n}\left(\bar{A}_{i}^{T}\left(P_{i}+P_{i}^{T}\right)\left(R B_{i}-\sum_{j=1}^{n} p_{j} R B_{j}\right)\right)}{\left.2 \sum_{i=1}^{n}\left(\left(R B_{i}-\sum_{j=1}^{n} p_{j} R B_{j}\right)^{T} P_{i}\left(R B_{i}-\sum_{j=1}^{n} p_{j} R B_{j}\right)\right)\right)^{2}}\right)^{T}\left(R B_{i}-\sum_{j=1}^{n} p_{j} R B_{j}\right)\right) \\
& \times \sum_{i=1}^{n}\left(\left(R B_{i}-\sum_{j=1}^{n} p_{j} R B_{j}\right)^{T} P_{i}(R\right.
\end{aligned}
$$

thus

$$
L(\mathrm{R})=\sum_{i=1}^{n}\left(\bar{A}_{i}^{T} P_{i} \bar{A}_{i}\right)-\frac{\left(\sum_{i=1}^{n}\left(\bar{A}_{i}^{T}\left(P_{i}+P_{i}^{T}\right)\left(R B_{i}-\sum_{j=1}^{n} p_{j} R B_{j}\right)\right)\right)^{2}}{4 \sum_{i=1}^{n}\left(\left(R B_{i}-\sum_{j=1}^{n} p_{j} R B_{j}\right)^{T} P_{i}\left(R B_{i}-\sum_{j=1}^{n} p_{j} R B_{j}\right)\right)} .
$$

Substituting Eq. (18) into Eq. (6), one gets a very complex equation from which the analytical formula of $\mathrm{R}$ is impossible. In other words, the analytical solution of $\mathrm{R}$ does not exist when the rotation is non-isotropic and even correlated in different coordinate direction. Therefore, to overcome the problem, two kinds of iterative approaches are 
presented in this paper. One is dependent on the objective function value, while the other is dependent on the derivative of function.

\section{Iterative approach dependent on function value}

Iterative approach dependent on function value is a family of iterative approach, also called direct search method that does not depend on the derivative information of function. It is very useful in the cases that the deduce process of derivative of function is very difficult and even derivative of function is not existing. The direct search concept is firstly presented by Hooke and Jeeves (1961). Pattern search method is a kind of very popular direct search method, and for its more detail, the readers are referred to e.g. Torczon (1997), Lewis, et al. (2000), Dolan, et al. (2003), Al-Sumaita, et al. (2007), Zeng and Yi (2010b). In this paper pattern search method is supposed to solve the Lagrangian minimum problem of Eq. (18).

The variable in Eq. (18) namely $\mathrm{R}$ is a matrix with 3 rows and 3 columns, usually represented by rotation angles (see e.g. El-Habiby et al. 2009; Zeng and Yi, 2011), unit quaternion (see e.g. Shen et al. 2006; Zeng and Yi 2011), and Rodrigues matrix (see e.g. Zeng and Yi 2010a). If the form of rotation matrix represented by rotation angles is used in searching the solution of the minimum problem, there are a large number of computations of trigonometric function, which slows down the speed of computation and even leads to a failure of iterative computation. If the form of rotation matrix represented by unit quaternion is used, there are four variables and a constraint condition that the sum of squares of variables equals to 1 . Searching the solution of the minimum problem in this case is more difficult than that in the case which has three variables space and is unconstrained. So the form of rotation matrix represented by Rodrigues matrix is adopted for its simple algebraic computation.

Rodrigues matrix can be written as

$$
R=(I+r)(I-r)^{-1},
$$

where $r$ is an anti-symmetric matrix formed with a Gibbs vector $v=\left(\begin{array}{lll}v_{x} & v_{y} & v_{z}\end{array}\right)$ as

$$
r=\left[\begin{array}{ccc}
0 & -v_{z} & -v_{y} \\
v_{z} & 0 & -v_{x} \\
v_{y} & v_{x} & 0
\end{array}\right] .
$$

\section{Iterative approach dependent on derivatives}

Substituting Eq. (11) into Eq. (1) and utilizing Eq. (13), one gets the 3D datum transformation model that eliminates the translation parameters. The form of rotation matrix represented by Rodrigues matrix is adopted with the same consideration as Sect. 3.

$$
\bar{A}_{i}=\lambda R B_{i}-\lambda \sum_{j=1}^{n} p_{j} R B_{j} .
$$


Carrying out the Linearization of Eq. (21), one gets

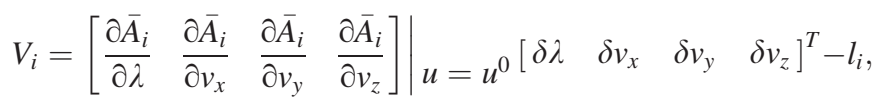

where

$$
u=\left[\begin{array}{llll}
\lambda & v_{x} & v_{y} & v_{z}
\end{array}\right]^{T}
$$

is the unknown, $u^{0}$ is its approximate value and

$$
\delta u=\left[\begin{array}{llll}
\delta \lambda & \delta v_{x} & \delta v_{y} & \delta v_{z}
\end{array}\right]^{T}
$$

is the correction of the unknown.

$$
\begin{gathered}
\frac{\partial \bar{A}_{i}}{\partial \lambda}=R B_{i}-\sum_{j=1}^{n} p_{j} R B_{j}, \\
\frac{\partial \bar{A}_{i}}{\partial v_{x}}=\lambda \frac{\partial R}{\partial v_{x}} B_{i}-\lambda \sum_{j=1}^{n} p_{j} \frac{\partial R}{\partial v_{x}} B_{j}, \\
\frac{\partial \bar{A}_{i}}{\partial v_{y}}=\lambda \frac{\partial R}{\partial v_{y}} B_{i}-\lambda \sum_{j=1}^{n} p_{j} \frac{\partial R}{\partial v_{y}} B_{j}, \\
\frac{\partial \bar{A}_{i}}{\partial v_{z}}=\lambda \frac{\partial R}{\partial v_{z}} B_{i}-\lambda \sum_{j=1}^{n} p_{j} \frac{\partial R}{\partial v_{z}} B_{j}, \\
l_{i}=\bar{A}_{i}-\lambda R B_{i}+\lambda \sum_{j=1}^{n} p_{j} R B_{j},
\end{gathered}
$$

and

$$
\begin{aligned}
& \frac{\partial R}{\partial v_{x}}=\frac{\partial r}{\partial v_{x}}(I-r)^{-1}+(I+r)(I-r)^{-1} \frac{\partial r}{\partial v_{x}}(I-r)^{-1} \\
&=\frac{\partial r}{\partial v_{x}}(I-r)^{-1}+R \frac{\partial r}{\partial v_{x}}(I-r)^{-1} \\
&=(I+R) \frac{\partial r}{\partial v_{x}}(I-r)^{-1} \\
& \frac{\partial r}{\partial v_{x}}=\left[\begin{array}{ccc}
0 & 0 & 0 \\
0 & 0 & -1 \\
0 & 1 & 0
\end{array}\right] .
\end{aligned}
$$

Similarly

$$
\frac{\partial R}{\partial v_{y}}=(I+R) \frac{\partial r}{\partial v_{y}}(I-r)^{-1}
$$




$$
\begin{gathered}
\frac{\partial r}{\partial v_{y}}=\left[\begin{array}{ccc}
0 & 0 & -1 \\
0 & 0 & 0 \\
1 & 0 & 0
\end{array}\right], \\
\frac{\partial R}{\partial v_{z}}=(I+R) \frac{\partial r}{\partial v_{z}}(I-r)^{-1}, \\
\frac{\partial r}{\partial v_{z}}=\left[\begin{array}{ccc}
0 & -1 & 0 \\
1 & 0 & 0 \\
0 & 0 & 0
\end{array}\right] .
\end{gathered}
$$

Let

$$
M_{i}=\left.\left[\begin{array}{llll}
\frac{\partial \bar{A}_{i}}{\partial \lambda} & \frac{\partial \bar{A}_{i}}{\partial v_{x}} & \frac{\partial \bar{A}_{i}}{\partial v_{y}} & \frac{\partial \bar{A}_{i}}{\partial v_{z}}
\end{array}\right]\right|_{u=u^{0}}
$$

and then Eq. (22) can be written as

$$
V_{i}=M_{i} \delta u-l_{i}
$$

Further let

$$
\begin{gathered}
V=\left[\begin{array}{llll}
V_{1} & V_{2} & \cdots & V_{n}
\end{array}\right]^{T}, \\
M=\left[\begin{array}{llll}
M_{1}^{T} & M_{2}^{T} & \cdots & M_{n}^{T}
\end{array}\right]^{T}, \\
l=\left[\begin{array}{llll}
l_{1} & l_{2} & \cdots & l_{n}
\end{array}\right]^{T},
\end{gathered}
$$

thus

$$
V=M \delta u-l \text {. }
$$

According to the least squares, the solution is

$$
\delta u=\left(M^{T} P M\right)^{-1} M^{T} P l,
$$

where

$$
P=\left[\begin{array}{llll}
P_{1} & & & \\
& P_{2} & & \\
& & \ddots & \\
& & & P_{n}
\end{array}\right] .
$$

Because the approximation of the unknown is usually tough, iterative computations are needed, i.e., one firstly gives the approximation $u^{0}$, then solve the correction $\delta u$ by the above approach, next give the approximation of next iteration as $u^{0}+\delta u$, and then repeat the above operation until every element of $\delta u$ is less than a given tolerance.

\section{Numerical experiments and discussion}

The experimental data is adopted from Grafarend and Awange (2003). The coordinates of 7 control points in the system B (local system) and A (WGS-84 system) are listed in Tables 1 and 2. 
Table 1 Coordinates and variances in system B (local system)

\begin{tabular}{lllllllll}
\hline Station name & \multicolumn{2}{l}{ System B (local system) $(\mathrm{m})$} & & \multicolumn{5}{l}{ Variance $\left(\mathrm{m}^{2}\right)$} \\
\cline { 2 - 3 } \cline { 7 - 8 } & $x$ & $y$ & & & & $\sigma_{x}^{2}$ & $\sigma_{y}^{2}$ & $\sigma_{z}^{2}$ \\
\hline Solitude & $4,157,222.543$ & $664,789.307$ & $4,774,952.099$ & & 0.14330000 & 0.16126012 & 0.25780367 \\
Buoch Zeil & $4,149,043.336$ & $688,836.443$ & $4,778,632.188$ & & 0.15510000 & 0.19971861 & 0.18798665 \\
Hohenneuffen & $4,172,803.511$ & $690,340.078$ & $4,758,129.701$ & & 0.15030000 & 0.12979970 & 0.18605368 \\
Kuehlenberg & $4,177,148.376$ & $642,997.635$ & $4,760,764.800$ & & 0.14000000 & 0.24000544 & 0.12159004 \\
Ex Mergelaec & $4,137,012.190$ & $671,808.029$ & $4,791,128.215$ & & 0.14590000 & 0.14040930 & 0.20278356 \\
Ex Hof Asperg & $4,146,292.729$ & $666,952.887$ & $4,783,859.856$ & & 0.14690000 & 0.19497925 & 0.15982531 \\
Ex Kaisersbach & $4,138,759.902$ & $702,670.738$ & $4,785,552.196$ & & 0.122000000 & 0.14330598 & 0.17612573 \\
\hline
\end{tabular}

Table 2 Coordinates and variances in system A (WGS-84)

\begin{tabular}{lllllllll}
\hline Station name & \multicolumn{5}{l}{ System A (WGS-84) $(\mathrm{m})$} & & \multicolumn{5}{l}{ Variance $\left(\mathrm{m}^{2}\right)$} \\
\cline { 2 - 3 } & $X$ & $Y$ & $Z$ & & $\sigma_{X}^{2}$ & $\sigma_{Y}^{2}$ & $\sigma_{Z}^{2}$ \\
\hline Solitude & $4,157,870.237$ & $664,818.678$ & $4,775,416.524$ & & 0.01030000 & 0.01625193 & 0.02285900 \\
Buoch Zeil & $4,149,691.049$ & $688,865.785$ & $4,779,096.588$ & & 0.00380000 & 0.00395319 & 0.00737509 \\
Hohenneuffen & $4,173,451.354$ & $690,369.375$ & $4,758,594.075$ & & 0.00060000 & 0.00100625 & 0.00156868 \\
Kuehlenberg & $4,177,796.064$ & $643,026.700$ & $4,761,228.899$ & & 0.01140000 & 0.01788546 & 0.02858824 \\
Ex Mergelaec & $4,137,659.549$ & $671,837.337$ & $4,791,592.531$ & & 0.00680000 & 0.00506161 & 0.01762072 \\
Ex Hof Asperg & $4,146,940.228$ & $666,982.151$ & $4,784,324.099$ & & 0.00002000 & 0.00001245 & 0.00002713 \\
Ex Kaisersbach & $4,139,407.506$ & $702,700.227$ & $4,786,016.645$ & & 0.00410000 & 0.00458562 & 0.00975938 \\
\hline
\end{tabular}

The numerical experiments have two parts. One is the special case that the weight matrix is identity one. The other is the common case of arbitrary form of weight matrix. It is supposed in the common case that the precisions of three coordinate directions in the local system are consistent, and then $\sigma_{x}^{2}$ adopts the same value as Grafarend and Awange (2003), and $\sigma_{y}^{2}, \sigma_{z}^{2}$ are generated with 'randn' command in Matlab. Additionally, the precisions of three coordinate directions in the WGS-84 system are inconsistent, namely the $X$ and $Y$ coordinates have the same precisions, but $Z$ coordinates has lower precisions than planar $X$ and $Y$ coordinates, and here $\sigma_{Z}^{2}=2 \sigma_{X}^{2}$ is assumed. $\sigma_{X}^{2}$ adopts the same value as Grafarend and Awange (2003), and $\sigma_{Y}^{2}, \sigma_{Z}^{2}$ are generated with 'randn' command in Matlab. The correlation of each point is not taken into account. The generated variances are listed in Tables 1 and 2. The weight matrix is generated with the same method as Grafarend and Awange (2003), and the result is list in Table 3.

The iterative approach dependent on function value and iterative approach dependent on derivatives are employed to recover the 7 transformation parameters. In the process of the former, the initial value of $v$ is set as $\left(\begin{array}{lll}0 & 0 & 0\end{array}\right)$, the stopping criteria is that the mesh tolerance or function tolerance is less than $10^{-12}$, and through 84 iterative times for the special case and 82 iterative times for the common case, the iterations are both convergent and the results are listed in Tables 4, 5, 6 and 7 respectively. In the process of the latter, the approximation of the unknown value $u$ is set as $\left(\begin{array}{llll}1 & 0 & 0 & 0\end{array}\right)$, the stopping criteria is that every element of $\delta u$ is less than $10^{-12}$, and through both 2 iterative times for the special case and the common case, the iterations are both 
Table 3 Weight matrix

\begin{tabular}{lrrrlrrr}
\hline $\begin{array}{l}\text { Weight } \\
\text { matrix }\end{array}$ & \multicolumn{1}{c}{ Values } & \multicolumn{5}{c}{$\begin{array}{l}\text { Weight } \\
\text { matrix }\end{array}$} \\
\hline P1 & 6.51034885 & 0.00005093 & -0.00004319 & P5 & 6.54871861 & 0.00005901 & -0.00004779 \\
& 0.00005093 & 5.63336324 & 0.00000938 & & 0.00005901 & 6.87415224 & 0.00000942 \\
& -0.00004319 & 0.00000938 & 3.56295957 & & -0.00004779 & 0.00000942 & 4.53707026 \\
P2 & 6.29319762 & 0.00004981 & -0.00005080 & P6 & 6.80634928 & 0.00005418 & -0.00005986 \\
& 0.00004981 & 4.90980598 & -0.00000143 & & 0.00005418 & 5.12836620 & -0.00000546 \\
& -0.00005080 & -0.00000143 & 5.11865458 & & -0.00005986 & -0.00000546 & 6.25569978 \\
P3 & 6.62683154 & 0.00006515 & -0.00005464 & P7 & 7.93012846 & 0.00006479 & -0.00005873 \\
& 0.00006515 & 7.64482735 & 0.00001110 & & 0.00006479 & 6.76163613 & 0.00000578 \\
& -0.00005464 & 0.00001110 & 5.32979632 & & -0.00005873 & 0.00000578 & 5.37961018 \\
P4 & 6.60495162 & 0.00004390 & -0.00005242 & & & & \\
& 0.00004390 & 3.87756843 & -0.00001480 & & & & \\
& -0.00005242 & -0.00001480 & 6.65869204 & & & & \\
\end{tabular}

Table 4 Result of 3D datum transformation in the special case

\begin{tabular}{|c|c|c|c|c|c|c|}
\hline & Iteration based & on function va & & Iteration based & & \\
\hline $\begin{array}{c}\text { Iterative } \\
\text { times }\end{array}$ & 84 & & & 2 & & \\
\hline$v$ & 0.0000024308 & 0.0000021681 & -0.0000024065 & 0.0000024204 & 0.0000021664 & -0.0000024073 \\
\hline$R$ & 1.0000000000 & 0.0000048131 & -0.0000043362 & 1.0000000000 & 0.0000048146 & -0.0000043328 \\
\hline & -0.0000048131 & 1.0000000000 & -0.0000048615 & -0.0000048146 & 1.0000000000 & -0.0000048409 \\
\hline & 0.0000043362 & 0.0000048615 & 1.0000000000 & 0.0000043327 & 0.0000048409 & 1.0000000000 \\
\hline$\Delta X(\mathrm{~m})$ & 641.8981 & & & 641.8804 & & \\
\hline$\Delta Y(\mathrm{~m})$ & 68.7474 & & & 68.6553 & & \\
\hline$\Delta Z(\mathrm{~m})$ & 416.3697 & & & 416.3982 & & \\
\hline$\lambda$ & 1. 000005583 & & & 1. 000005583 & & \\
\hline
\end{tabular}

Table 5 Transformation residuals in the special case

\begin{tabular}{|c|c|c|c|c|c|c|}
\hline \multirow[t]{2}{*}{ Station name } & \multicolumn{3}{|c|}{ Iteration based on function value (m) } & \multicolumn{3}{|c|}{ Iteration based on derivative (m) } \\
\hline & $X$ & $Y$ & $Z$ & $X$ & $Y$ & $Z$ \\
\hline Solitude & 0.0940 & 0.1351 & 0.1404 & 0.0940 & 0.1351 & 0.1402 \\
\hline Buoch Zeil & 0.0588 & -0.0496 & 0.0134 & 0.0588 & -0.0497 & 0.0137 \\
\hline Hohenneuffen & -0.0399 & -0.0883 & -0.0084 & -0.0399 & -0.0879 & -0.0081 \\
\hline Kuehlenberg & 0.0201 & -0.0223 & -0.0868 & 0.0202 & -0.0220 & -0.0874 \\
\hline Ex Mergelaec & -0.0918 & 0.0143 & -0.0054 & -0.0919 & 0.0139 & -0.0055 \\
\hline Ex Hof Asperg & -0.0118 & 0.0067 & -0.0544 & -0.0118 & 0.0065 & -0.0546 \\
\hline Ex Kaisersbach & -0.0293 & 0.0043 & 0.0012 & -0.0294 & 0.0041 & 0.0017 \\
\hline$\sigma_{m}(\mathrm{~m})$ & 0.0773 & & & 0.0773 & & \\
\hline
\end{tabular}


Table 6 Result of 3D datum transformation in the common case

\begin{tabular}{|c|c|c|c|c|c|c|}
\hline & \multicolumn{3}{|c|}{ Iteration based on function value } & \multicolumn{3}{|l|}{$\begin{array}{l}\text { Iteration based } \\
\text { on derivative }\end{array}$} \\
\hline $\begin{array}{c}\text { Iterative } \\
\text { times }\end{array}$ & 82 & & & 2 & & \\
\hline$v$ & 0.0000025108 & 0.0000021756 & -0.0000024401 & 0.0000025040 & 0.0000021746 & -0.0000024332 \\
\hline \multirow[t]{3}{*}{$R$} & 1.0000000000 & 0.0000048801 & -0.0000043512 & 1.0000000000 & 0.0000048664 & -0.0000043491 \\
\hline & -0.0000048801 & 1.0000000000 & -0.0000050217 & -0.0000048664 & 1.0000000000 & -0.0000050079 \\
\hline & 0.0000043511 & 0.0000050217 & 1.0000000000 & 0.0000043491 & 0.0000050080 & 1.0000000000 \\
\hline$\Delta X(\mathrm{~m})$ & 641.8754 & & & 641.8770 & & \\
\hline$\Delta Y(\mathrm{~m})$ & 69.7813 & & & 69.6590 & & \\
\hline$\Delta Z(\mathrm{~m})$ & 416.1342 & & & 416.1543 & & \\
\hline$\lambda$ & 1.000005594 & & & 1.000005593 & & \\
\hline
\end{tabular}

Table 7 Transformation residuals in the common case

\begin{tabular}{|c|c|c|c|c|c|c|}
\hline \multirow[t]{2}{*}{ Station name } & \multicolumn{3}{|c|}{ Iteration based on function value $(\mathrm{m})$} & \multicolumn{3}{|c|}{ Iteration based on derivative $(\mathrm{m})$} \\
\hline & $X$ & $Y$ & $Z$ & $X$ & $Y$ & $Z$ \\
\hline Solitude & 0.0960 & 0.1373 & 0.1532 & 0.0959 & 0.1373 & 0.1530 \\
\hline Buoch Zeil & 0.0594 & -0.0477 & 0.0224 & 0.0596 & -0.0476 & 0.0226 \\
\hline Hohenneuffen & -0.0400 & -0.0881 & 0.0002 & -0.0398 & -0.0881 & 0.0004 \\
\hline Kuehlenberg & 0.0232 & -0.0208 & -0.0707 & 0.0228 & -0.0209 & -0.0711 \\
\hline Ex Mergelaec & -0.0898 & 0.0176 & 0.0064 & -0.0899 & 0.0177 & 0.0063 \\
\hline Ex Hof Asperg & -0.0096 & 0.0096 & -0.0420 & -0.0098 & 0.0096 & -0.0421 \\
\hline Ex Kaisersbach & -0.0294 & 0.0065 & 0.0080 & -0.0291 & 0.0066 & 0.0083 \\
\hline$\sigma_{m}(\mathrm{~m})$ & 0.1830 & & & 0.1830 & & \\
\hline
\end{tabular}

convergent and the results are listed in Tables 4, 5, 6 and 7 respectively. $\sigma_{m}$ in Tables 5 and 7 is mean error and computed by

$$
\sigma_{m}=\sqrt{\frac{L}{3 n-7}}
$$

and $L$ is the Lagrangian extremum by Eq. (1) or (18).

Comparing the Table 4 and the result of Grafarend and Awange (2003) namely I-LESS procrustes algorithm, it is seen that the result of iterative approach based on derivatives is identical to that of I-LESS procrustes algorithm. It is worthy to be noted that there is a little mistake in Grafarend and Awange (2003) that the second element and third element in the first row of rotation matrix wrongly exchange position. In addition the result of iterative approach dependent on function value is slightly different from that of I-LESS procrustes algorithm, and the bias of translation parameter is $\mathrm{cm}$ level. However, from Table 5, it is seen that the transformation residual is in sub-mm level.

It is seen from Table 6 that the result of iterative approach dependent on function value is slightly different from that of iterative approach based on derivatives, and the bias of translation parameter is dm level. However, from Table 7 it is seen that the transformation residual is in sub-mm level. 
Fig. 1 Iterative process based on function value

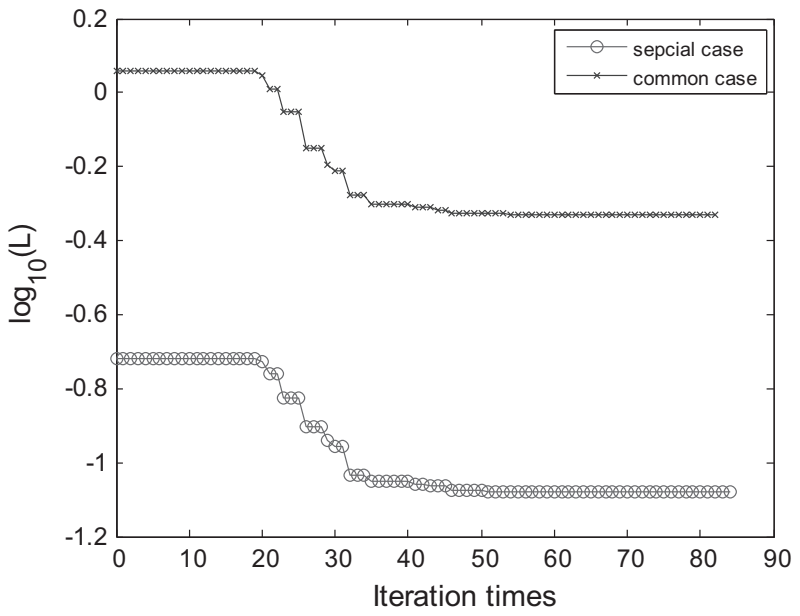

In order to investigate the performance of iterative approach dependent on function value namely pattern search method, the iterative process is drawn in Fig. 1. It shows that the convergent process is highly similar although the weight matrix is different for the two cases. And the approach is stable and feasible.

\section{Conclusion}

Through lots of derivation of this paper, it is found that analytical solution of 3D datum transformation does not exist in the case that the weight matrix is non-isotropic and even correlated in different coordinate direction. Thus the iterative approach of $3 \mathrm{D}$ datum transformation is investigated. The paper presents two kinds of iterative approach, i.e. iterative approach dependent on the objective function value and iterative approach dependent on the derivative of function. In order to improve the speed and reliability, the form of rotation matrix represented by Rodrigues matrix rather than rotation angles or unit quaternion is adopted for both two iterative approaches.

The numerical experiment and analysis shows the presented two kinds of iterative approach are both correct and efficient. Further it is thought that iterative approach dependent on function value is convenient for its objective function can be established easily and fast, however it needs a great deal of iterative computation, therefore it is slower than the iterative approach based on derivatives, and its result is slightly worse than the iterative approach based on derivatives. On the other hand, the iterative approach based on derivatives has lots of work to deduce derivatives, but once the model is established, the iterative computation is faster and more reliable than the iterative approach dependent on function value. From the view of computing speed and reliability, the iterative approach based on derivatives is preferred.

Acknowledgments The study is supported jointly by the Open Foundation of Hubei Key Laboratory of Construction and Management in Hydropower Engineering, China Three Gorges University (Grant No. 2014KSD13), the 2015 Open Foundation of Hubei Key Laboratory of Intelligent Vision Based Monitoring for Hydroelectric Engineering, China Three Gorges University (Grant No. 2015KLA06), the Open Research Fund Program of the Key Laboratory of Geospace Environment and Geodesy, Ministry of Education, China (Grant No. 11-01-04), the Open Foundation of the Key Laboratory of Precise Engineering and Industry 
Surveying, National Administration of Surveying, Mapping and Geoinformation of China (Grant No. PF2011-4), and National Natural Science Foundation of China (Grant No. 41104009). The first author is grateful for the support and good working atmosphere provided by his research team in China Three Gorges University. The first author also thanks two anonymous reviewers for valuable comments and suggestions, which enhanced the quality of this manuscript.

\section{References}

Aktuğ B (2012) Weakly multicollinear datum transformations. J Surv Eng 138(4):184-192

Akyilmaz O (2007) Total least squares solution of coordinate transformation. Surv Rev 39(303):68-80

Al-Sumaita JS, AL-Othmanb AK, Sykulski JK (2007) Application of pattern search method to power system valve-point economic load dispatch. Int J Electr Power Energy Syst 29(10):720-730

Burša M (1967) On the possibility of determining the rotation elements of geodetic reference systems on the basis of satellite observations. Stud Geophys Geod 11(4):390-396

Chen Y, Shen YZ, Liu DJ (2004) A simplified model of three dimensional-datum transformation adapted to big rotation angle. Geomat Inf Sci Wuhan Univ 29(12):1101-1105

Dermanis A (1998) Generalized inverses of nonlinear mappings and the nonlinear geodetic datum problem. J Geod 72(2):71-100

Dolan ED, Lewis RM, Torczon VJ (2003) On the local convergence of pattern search. SIAM J Optim 14(2):567-583. doi:10.1137/S1052623400374495

El-Habiby MM, Gao Y, Sideris MG (2009) Comparison and analysis of non-linear least squares methods for 3-D coordinates transformation. Surv Rev 41(311):26-43

El-Mowafy A, Fashir H, Al-Marzooqi Y (2009) Improved coordinate transformation in Dubai using a new interpolation approach of coordinate differences. Surv Rev 41(311):71-85

Ge Y, Yuan Y, Jia N (2013) More efficient methods among commonly used robust estimation methods for GPS coordinate transformation. Surv Rev 45(330):229-234

Grafarend EW, Awange JL (2003) Nonlinear analysis of the three-dimensional datum transformation [conformal group C7(3)]. J Geod 77:66-76

Han JY (2010) Noniterative approach for solving the indirect problems of linear reference frame transformations. J Surv Eng 136(4):150-156

Hooke R, Jeeves TA (1961) Direct search solution of numerical and statistical problems. J ACM $8(2): 212-229$

Kashani I (2006) Application of generalized approach to datum transformation between local classical and satellite-based geodetic networks. Surv Rev 38(299):412-422

Leick A (2004) GPS satellite surveying, 3rd edn. Wiley, Hoboken

Leick A, van Gelder BHW (1975) On similarity transformations and geodetic network distortions based on Doppler satellite observations. Report No. 235, Dept. of Geodetic Science, Ohio State Univ., Columbus, Ohio

Lewis RM, Torczon V, Trosset MW (2000) Direct search methods: then and now. J Comput Appl Math 124(1-2):191-207

Neitzel F (2010) Generalization of total least-squares on example of weighted 2D similarity transformation. J Geod 84(12):751-762. doi:10.1007/s00190-010-0408-0

Paláncz B, Awange JL, Völgyesi L (2013) Pareto optimality solution of the Gauss-Helmert model. Acta Geod Geophys 48:293-304

Shen YZ, Chen Y, Zheng DH (2006) A quaternion-based geodetic datum transformation algorithm. J Geod 80:233-239

Soler T, Snay RA (2004) Transforming positions and velocities between the International Terrestrial Reference Frame of 2000 and North American Datum of 1983. J Surv Eng 130(2):49-55

Soycan M, Soycan A (2008) Transformation of 3D GPS Cartesian coordinates to ED50 using polynomial fitting by robust re-weighting technique. Surv Rev 40(308):142-155

Torczon V (1997) On the convergence of pattern search algorithms. SIAM J Optim 7:1-25

Watson GA (2006) Computing Helmert transformations. J Comput Appl Math 197(2):387-394. doi:10. 1016/j.cam.2005.06.047

Zeng HE (2014) Planar coordinate transformation and its parameter estimation in the complex number field. Acta Geod Geophys 49(1):79-94

Zeng HE (2015) Analytical algorithm of weighted 3D datum transformation using the constraint of orthonormal matrix. Earth, Planets Space 67:105 
Zeng HE, Huang SX (2008) A kind of direct search method adopted to solve 3D coordinate transformation parameters. Geomat Inf Sci Wuhan Univ 33(11):1118-1121

Zeng WX, Tao BZ (2003) Non-linear adjustment model of three-dimensional coordinate transformation. Geomat Inf Sci Wuhan Univ 28(5):566-568

Zeng HE, Yi QL (2010a) A new analytical solution of nonlinear geodetic datum transformation. In: Proceedings of the 18th international conference on geoinformatics. doi:10.1109/GEOINFORMATICS. 2010.5568048

Zeng HE, Yi QL (2010b) A solution to space resection based on pattern search method. In: Proceedings of the 18th international conference on geoinformatics. doi:10.1109/GEOINFORMATICS.2010.5567847

Zeng HE, Yi QL (2011) Quaternion-based iterative solution of three-dimensional coordinate transformation problem. J Comput 6(7):1361-1368 